\title{
Vibrational Properties of Boron-Nitride Nanotubes: Effects of Finite Length and Bundling
}

\author{
Ludger Wirtz and Angel Rubio
}

\begin{abstract}
We present ab initio calculations of phonons in single-wall boron-nitride (BN) nanotubes. Raman and infrared (IR) active modes of isolated and infinitely long tubes are evaluated according to the nonsymmorphic rod groups of $\mathrm{BN}$ nanotubes. For tubes of finite length, the selection rules are less restrictive and give rise to additional modes, which may be observed in Raman and IR spectroscopy with an intensity depending on the tube length. Bundling of tubes is shown to have little effect on the phonon frequencies. However, arranging tubes in a large periodic array (larger than the wavelength of incoming light) gives rise to a strong frequency shift (longitudinal-optical-transverse-optical splitting) of certain modes due to the establishing of a macroscopic electric field. Modes of $A_{1}$ symmetry experience a shift for laser light along the tube axis and $E_{1}$ modes are split for light incidence in the perpendicular direction.
\end{abstract}

Index Terms-Boron-nitride (BN) nanotubes, infrared (IR) spectroscopy, phonons, Raman spectroscopy.

\section{INTRODUCTION}

B ORON nitride (BN) is isoelectronic to carbon and displays-among others-a graphite-like hexagonal phase (h-BN), which has been extensively studied by various spectroscopic methods [1]-[6]. The elastic constants are very similar (although smaller), but the polar nature of the BN bond leads to significant changes in the electronic structure of $\mathrm{h}-\mathrm{BN}$ as compared to graphite. While graphite is a semimetal (zero bandgap in the single sheet), h-BN has a large bandgap of approximately $5.5 \mathrm{eV}$ [3]. Furthermore, its high thermal stability and relative chemical inertness distinguishes it from its carbon counterpart.

The structure of a carbon nanotube can be explained by rolling up a single graphene sheet. It is natural to do the same with a single hexagonal BN sheet and construct a BN nanotube, which is isoelectronic to $\mathrm{C}$ nanotubes, but should carry over some of the characteristic differences of h-BN with respect to graphite. Indeed, tight-binding [7] and first-principles calculations [8] demonstrated that BN tubes are stable and have a bandgap similar to h-BN, independent of the tube diameter and chirality. Furthermore, the bandgap is independent of the

Manuscript received July 31, 2003; revised August 7, 2003. This work was supported in part by the European Union under Grant HPRN-CT-2000-00 128 , and in part by the Spanish Ministry of Science and Technology under Grant MAT2001-0946, and in part by the University of the Basque Country unde Contract 00 206.215-13 639/2001. This paper was presented in part at the Symposium of Microtechnologies for the New Millennium, Nanotechnology Conference, Gran-Canaria, Spain, May 2003

The authors are with the Donostia International Physics Center and Department of Material Physics, University of the Basque Country, 20018 Donostia-San Sebastián, Spain (e-mail: 1wirtz@sc.ehu.es).

Digital Object Identifier 10.1109/TNANO.2003.820511 number of walls in multiwall nanotubes as long as the inner diameter is above $0.8 \mathrm{~nm}$.

The first synthesis of multiwall $\mathrm{BN}$ tubes was reported in 1995 [9]. By now, BN tubes are routinely produced in several groups [10]-[16]. The production of single-wall BN tubes in gram quantities [17] is a further milestone toward applications of BN nanotubes. The as-produced samples of BN nanotubes face the same problems as its carbon brethren. The raw product at the end of the production process contains tubes of various lengths, diameters, and chiralities, some of which are isolated while others form bundles. This tube material may furthermore be contaminated by catalyst particles and raw material from the beginning of the production process. Further processing at this stage requires detailed information from spectroscopy.

Several spectroscopic methods are commonly used for the identification and characterization of nanotube samples. High-resolution transmission electron (HRTEM) allows for a quick view at the scene with almost atomic resolution. Scanning tunneling microscopy/spectroscopy (STM/STS) allow to get atomic resolution and to map the electronic structure to the underlying nanotube geometry [18]. In optical spectroscopy, using laser light, the spacial resolution is lost. However, alternative information about the band structure and the vibrational properties of the constituents can be gained. Optical absorption spectroscopy probes the electronic band structure by direct excitation of an electron from the valence to the conduction band. Since BN tubes have a wide bandgap, either multiphoton processes or UV light are necessary for this process to occur in BN nanotubes. Complementary information about the band structure of multiwall BNnanotubes has recently been obtained by electron energy loss spectroscopy (EELS) [19].

Recent spectrofluorimetric data on carbon single-wall nanotubes (SWNT) suspended in an aqueous surfactant combined with Raman and optical absorption spectroscopy has been able to provide information about tube chirality [20]. At lower energy, infrared (IR) absorption probes the direct excitation of phonons. Finally, Raman spectroscopy probes the excitation of phonons by measuring the frequency shift in elastically scattered laser light.

We investigate the application of the latter two spectroscopic methods (i.e., Raman and IR) for the characterization of BN nanotubes. For carbon materials, Raman scattering is resonantly enhanced because the bandgap is zero or very small and electrons can be excited from the valence to the conduction band. This results in a high intensity of the spectra and a sensitive dependence on the laser frequency. Therefore, Raman STS has become an accurate highly diameter-selective tool for the characterization of carbon nanotubes [21], [22]. In BN tubes, 
Raman scattering is nonresonant due to the large bandgap of the tubes. The resulting spectra are, therefore, weaker in intensity and must be carefully separated from a possible overlap by resonant Raman scattering from contaminants [23]. On the other hand, in BN tubes, the efficiency of IR-absorption is enhanced by the polarity of the material and gives rise to a much more pronounced IR spectrum [24] than in the case of carbon tubes where the IR spectra have very little structure and can hardly be distinguished from the IR spectrum of graphite [25].

For the interpretation of measured spectra [23], an accurate knowledge of the phonon frequencies as a function of tube diameter and chirality is indispensable. In a recent paper [26], we have presented $a b$ initio calculations of phonons in ideal isolated infinitely long $\mathrm{BN}$ nanotubes. In this paper, the emphasis lies on the effects of deviation from this ideal case, i.e., finite tube size and occurrence of tubes in bundles. Experimentally produced tubes have a finite length (typically between 200-400 $\mathrm{nm}$ [17]), which is shorter than the wavelength of IR light. In this case, the tubes appear as very large molecules rather than infinitely extended. This lowers the nonsymmorphic rod group symmetry of the tubes [27]-[29] to the point-group symmetry of a finite-size tube. Lower symmetry leads to a relaxation of selection rules and gives rise to additional "allowed" Raman and IR active modes. The effect of bundling of tubes is difficult to treat exactly because a super cell for a bundle of, e.g., ten tubes would be prohibitively large. As an approximation to the bundling effect, we study, therefore, the phonon frequencies in an (infinitely extended) closely packed array of tubes. In this case, the polarity of $\mathrm{BN}$ allows for the coupling of certain modes to a self-induced macroscopic electric field (Lydanne-Sachs-Teller splitting [30]), which leads to a shift of some frequencies. This effect depends on the direction of the incoming laser light.

The structure of this paper is as follows. In Section II, we give some details on the calculation of phonon frequencies by density-functional perturbation theory (DFPT) and compare with other more empirical methods. The results for ideal infinitely long tubes are summarized in Section III where an intuitive explanation of the symmetry of Raman and IR active modes is also given. In Section IV, we present the frequencies of Raman and IR active phonon modes in tubes of finite length. In Section V, we discuss the expected Raman and IR spectra in a periodic array of densely packed tubes.

\section{Method of CAlculation}

The phonon frequencies $\omega$ as a function of the phonon wave vector $q$ are the solution of the secular equation

$$
\operatorname{det}\left|\frac{1}{\sqrt{M_{s} M_{t}}} C_{s t}^{\alpha \beta}(q)-\omega^{2}(q)\right|=0 .
$$

$M_{s}$ and $M_{t}$ denote the atomic masses of atoms $s$ and $t$, and the dynamical matrix is defined as

$$
C_{s t}^{\alpha \beta}(q)=\frac{\partial^{2} E}{\partial u_{s}^{* \alpha}(q) \partial u_{t}^{\beta}(q)}
$$

where $u_{s}^{\alpha}$ denotes the displacement of atom $s$ in direction $\alpha$. The second derivative of the energy in (2) corresponds to the change of the force acting on atom $t$ in direction $\beta$ with respect to a displacement of atom $s$ in direction $\alpha$ as follows:

$$
C_{s t}^{\alpha \beta}(q)=\frac{\partial}{\partial u_{s}^{* \alpha}(q)} F_{t}^{\beta}(q) .
$$

Note the dependence on $q$ of the dynamical matrix and displacements. In an explicit calculation of the dynamical matrix by displacing each of the atoms of the unit cell into all three directions, a periodic supercell has to be used, which is commensurate with the phonon wavelength $2 \pi / q$. Fourier transform of the $q$-dependent dynamical matrix leads to the real space force constant matrix $C_{s t}^{\alpha \beta}(R)$. A phonon calculation starts with a determination of the dynamical matrix in real space or reciprocal space (2).

Before discussing how the dynamical matrix is constructed for $\mathrm{BN}$ nanotubes, it is instructive to look at how phonons have been calculated in carbon tubes. The easiest, very intuitive, and computationally fast approach is the use of (real space) force constants that are fitted to experimentally measured phonon dispersion relations. A set of force constants up to fourth nearest neighbor interaction was originally used to describe the phonon dispersion relation of graphite and has also become the standard for nanotubes [31], [32]. However, the agreement between experiment and theory is limited to certain regions in the first Brillouin zone. Furthermore, recent ab initio calculations [33], which agree almost perfectly with the experimental dispersion relation of graphite, have explicitly listed force constants and demonstrated that the cutoff with the fourth nearest neighbor is questionable. A more precise, but also more costly, alternative to the use of a force constant approach is the explicit calculation of the dynamical matrix by tight binding (see, e.g., [34]) or $a b$ initio methods [33], [35]. For BN tubes, phonons have been calculated $a b$ initio [26] by tight-binding [36] and by a valence shell model [37].

We have chosen the ab initio approach for our calculation because the tight-binding calculations for phonons in $\mathrm{C}$ nanotubes produced deviations of up to $100 \mathrm{~cm}^{-1}$ for the phonons in the high-frequency regime. We use density-functional theory (DFT) in the local density approximation (LDA) as implemented in the code ABINIT. ${ }^{1}$ Only valence electrons are treated explicitly. Core electrons are described by Troullier-Martins pseudopotentials [38]. The wave functions are expanded in plane waves with an energy cutoff at 40 a.u. (atomic units). Since the code requires the use of a three-dimensional supercell, the (infinitely long) tubes are ordered in a triangular periodic array. In order to simulate an isolated tube, we choose a large inter-tube distance of 14 a.u. For the simulation of the "solid of tubes," a distance of 6.5 a.u., which corresponds roughly to the inter-sheet distance in h-BN is chosen. The tube geometry is optimized (until the forces acting on all atoms are smaller than $5 \times 10^{-5}$ a.u.) and the stress along the tube axis is minimized by adjusting the super-cell size along the tube axis.

The second derivatives in the dynamical matrix (2) are calculated in density-functional perturbation theory (DFPT) [39] as implemented in ABINIT [40]. This means that the atomic displacements are not performed explicitly, but treated as a per-

${ }^{1}$ The ABINIT code is a common project of the Universite Catholique de Louvain, Louvain, Switzerland, Corning Incorporated, and other contributors. [Online]. Available: http://www.abinit.org 
turbation. The equations for the first-order change in the electron density can be cast into a form similar as the Kohn-Sham equations [41] and must be solved self-consistently (i.e., adjusting the Hartree and the exchange-correlation potential with the change in density). In the case of (quasi-)isolated tubes, only the displacement perturbation of one boron and one nitrogen atom needs to be calculated explicitly. The residual elements of the dynamical matrix are obtained by symmetry transformation employing the nonsymmorphic rod group symmetry of the tubes. In the case of the close-packed tubes, the exact symmetry is lost and all atomic displacement have to be calculated.

Since $\mathrm{BN}$ is a polar material where $\mathrm{N}$ is slightly negatively charged and B is slightly positive, the long-range character of the Coulomb potential gives rise to a macroscopic electric field $E$ for longitudinal optical phonons in the limit $q \rightarrow 0$. The dynamical matrix (2) must, therefore, be corrected under inclusion of the Born effective charge tensor $Z_{s}^{* \alpha \beta}$ of the ions and the static dielectric tensor $\epsilon_{\infty}^{\alpha \beta}$. Both quantities can be calculated in DFPT [39], [40] from the macroscopic electric polarization $P$ of the medium through

$$
e Z_{s}^{* \alpha \beta}=\left.\Omega \frac{\partial P_{\alpha}}{\partial u_{s}^{\beta}(q=0)}\right|_{E=0}
$$

where $\Omega$ denotes the unit cell volume and

$$
\epsilon_{\infty}^{\alpha \beta}=\delta_{\alpha \beta}+\left.4 \pi \frac{\partial P_{\alpha}}{\partial E_{\beta}}\right|_{u_{s}(0)=0} .
$$

For (quasi-)one-dimensional systems like isolated tubes, no classical macroscopic electric field can be induced through longitudinal optical phonons and the above-mentioned corrections can be neglected. However, in Section V, it will be shown that the effect of electric-field corrections is very strong for some modes in large periodic arrays of tubes.

\section{RAMAN AND IR ACTIVE MODES IN INFINITELY LONG TUBES}

Since the photons of IR or visible light carry a momentum, which is negligible compared to the momentum associated with atomic motion, only phonons with a wave vector $q \approx 0$ (the " $\Gamma$ point" in reciprocal space) can be excited in first-order Raman and IR absorption processes. Selection rules impose a further restriction on the number of IR and Raman active modes. Only modes that transform under symmetry operations as a quadratic form are Raman active and only modes that transform as a vector are IR active. Higher symmetry leads to stricter selection rules and thereby to a lower number of active modes. Raman and IR active modes of carbon nanotubes have been frequently evaluated based on the point group symmetry of the unit cell [31]. However, Alon pointed out [27] that, for nanotube STS, the nonsymmorphic rod group symmetry of the tubes should be used. As in solid-state spectroscopy, where the "point group in the space group" rather than the point group of the unit cell determines the selection rules, it is the "point group in the rod group" that determines which modes are active in infinitely extended nanotubes. In Fig. 1, we demonstrate this for the case of
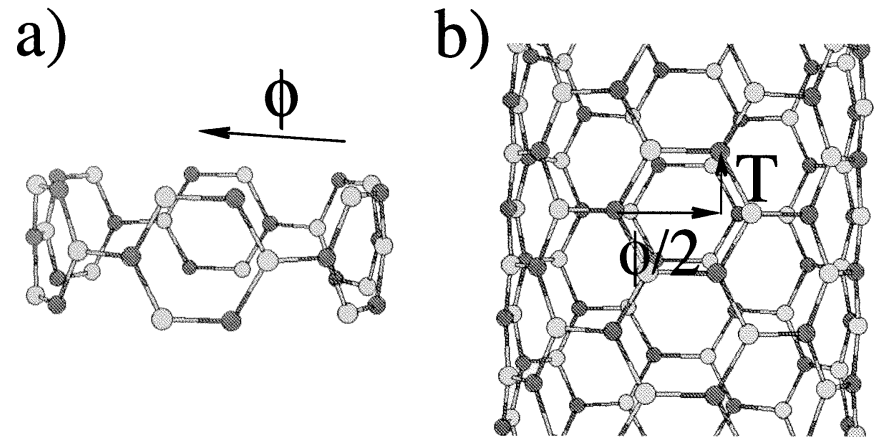

Fig. 1. Comparison of the point-group symmetry of the unit cell with the space-group symmetry of the armchair BN tubes.

TABLE I

SYMMETRIES AND NUMBER OF RAMAN AND IR ACTIVE MODES IN INFINITELY LONG BN NANOTUBES (FOLLOWING [27])

\begin{tabular}{l||l|l||l|l||l|l}
\hline \multicolumn{1}{c||}{} & \multicolumn{2}{c||}{\begin{tabular}{c} 
armchair $(n, n)$ \\
\multicolumn{2}{c||}{$C_{2 n h}$}
\end{tabular}} & \multicolumn{2}{c||}{$\begin{array}{c}\text { chiral }(n, m) \\
C_{N}\end{array}$} & \multicolumn{2}{c}{$\begin{array}{c}\text { zigzag }(n, 0) \\
C_{2 n v}\end{array}$} \\
\hline \hline Raman & $A_{g}$ & 3 & $A$ & 4 & $A_{1}$ & 3 \\
& $E_{1 g}$ & 2 & $E_{1}$ & 5 & $E_{1}$ & 5 \\
& $E_{2 g}$ & 4 & $E_{2}$ & 6 & $E_{2}$ & 6 \\
\hline IR & $A_{u}$ & 1 & $A$ & 4 & $A_{1}$ & 3 \\
& $E_{1 u}$ & 3 & $E_{1}$ & 5 & $E_{1}$ & 5 \\
\hline
\end{tabular}

an $(n, n)$ armchair tube. The left-hand side shows the unit cell of the tube containing $4 n$ atoms. (Note that the atoms at the lower and upper boundary of the unit cell are also contained in the adjacent unit cells and are, therefore, counted as "half" atoms.) It can be easily seen that the unit cell has an $n$-fold rotation axis (with rotation angle $\phi=2 \pi / n$ ) and a horizontal reflection symmetry plane. Therefore, the point-group symmetry of the unit cell is $C_{n h}$. The right-hand side of Fig. 1 demonstrates that, in the infinitely extended tube, rotation by $\phi / 2$ with a subsequent translation by the vector $T$ maps the system onto itself as well. The "point group in the rod group" of an armchair BN tube is, therefore, the $C_{2 n h}$ group. Table I summarizes the findings of [27] for the IR and Raman active modes in BN nanotubes. It lists the symmetry groups of armchair, chiral, and zigzag BN nanotubes, the symmetries of the active modes, and the corresponding number of modes with that symmetry. Note that for chiral and zigzag tubes, the IR active modes form a subset of the Raman active modes. This is different for armchair tubes, where the two sets are disjoint.

The symmetry of the active modes can be understood intuitively by applying the zone-folding method [31], i.e., rolling up a sheet into a tube. This is demonstrated for an armchair nanotube in Fig. 2. The left-hand side shows a part of a hexagonal sheet, which is rolled along the direction of $K_{\perp}$. The phonon wavevector along this direction will be quantized in the tubes. This corresponds to $2 n$ discrete values along the line $\Gamma \rightarrow M \rightarrow$ $\Gamma$ in the reciprocal space of the sheet (right-hand side of Fig. 2). The point $\mu=0$ corresponds to an $A$ mode of the tube where all equivalent atoms along the tube circumference move in phase. Fig. 3(a) demonstrates this for the radial buckling mode. (In the high-frequency modes, which are derived from the optical phonon branch of the sheet, $\mathrm{B}$ and $\mathrm{N}$ atoms oscillate with a phase difference of $\pi$, while in the low-frequency modes, e.g., the radial breathing mode, $\mathrm{B}$ and $\mathrm{N}$ atoms oscillate in phase.) 


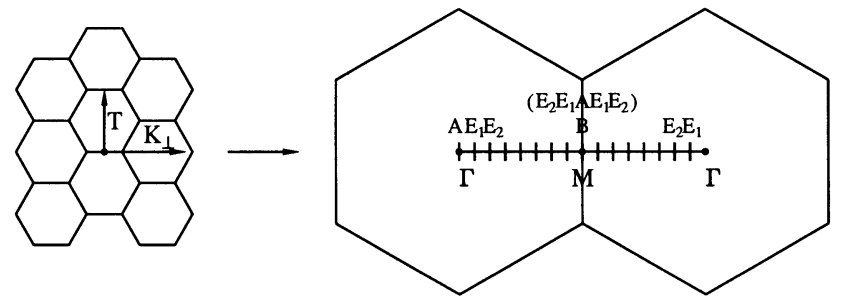

Fig. 2. Sketch of the zone-folding method for an $(n, n)$ armchair nanotubes. Left-hand side: hexagonal sheet with translation vector $T$ and perpendicular phonon wave vector $K_{\perp}$ along which the sheet is rolled into a tube. This corresponds to a quantization of $K_{\perp}$ in $2 n$ steps in the Brillouin zone of the sheet along the line $\Gamma \rightarrow \Gamma$ (right-hand side).

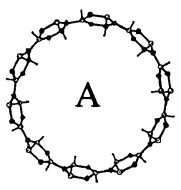

(a)

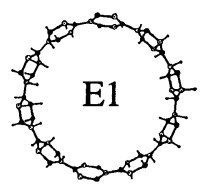

(b)

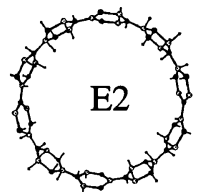

(c)

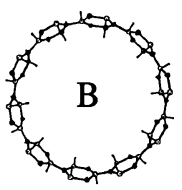

(d)
Fig. 3. Sketch of the $A, E_{1}, E_{2}$, and $B$ radial buckling modes.
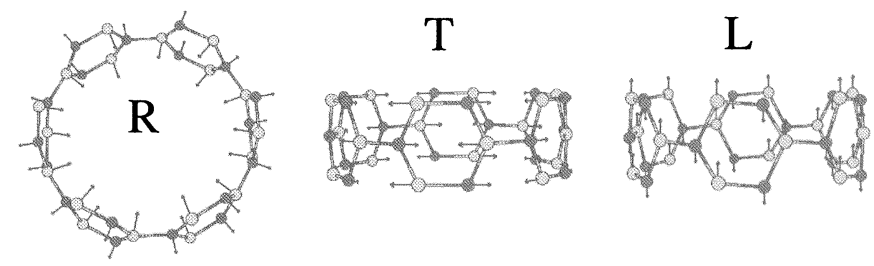

Fig. 4. High-frequency $A$ modes of a $(6,6)$ armchair nanotube: radial (R), tangential (L), and longitudinal (L) modes.

The points $\mu=1$ and $\mu=2 n-1$ in Fig. 2 are equivalent and lead to a doubly degenerate $E_{1}$ mode in the tube, which has two nodes along the circumference [see Fig. 3(b)]. Accordingly, the two points $\mu=2$ and $\mu=2 n-2$ correspond to a doubly degenerate $E_{2}$ mode with four nodes along the circumference [see Fig. 3(c)]. The construction of the modes $E_{3}, \ldots, E_{n-1}$ works analogously. The point $\mu=n$ finally leads to a nondegenerate $B$ mode where neighboring atoms of one kind oscillate with a phase difference of $\pi$ corresponding to a wave with $n-1$ nodes along the circumference, as depicted in Fig. 3(d). In Fig. 4, we show the $A$ modes of a $(6,6)$ armchair tube that are derived from the three optical modes of the sheet at $\Gamma$. The radial (R) buckling mode stems from the optical out-of-plane ( $\mathrm{ZO})$ mode of the sheet. The two optical in plane modes of the sheet [longitudinal optical (LO) and tangential optical (TO)] lead to a mode where the atoms oscillate in transverse-or tangential-(T) direction and a mode with oscillation in longitudinal (L) direction. From Fig. 4, it can also be seen that the $\mathrm{R}$ and $\mathrm{T}$ modes have even parity, i.e., they are mapped onto themselves under inversion at the center of the unit cell. The L mode, in contrast, has odd parity because the direction of atomic motion changes under inversion. For chiral and zigzag tubes, there is no inversion symmetry and the distinction between gerade and ungerade modes is absent. It is this presence/absence of inversion symmetry that leads to the above-noted fact that the IR active modes are also Raman active in chiral and zigzag tubes, while in armchair tubes, the two sets have no common elements.
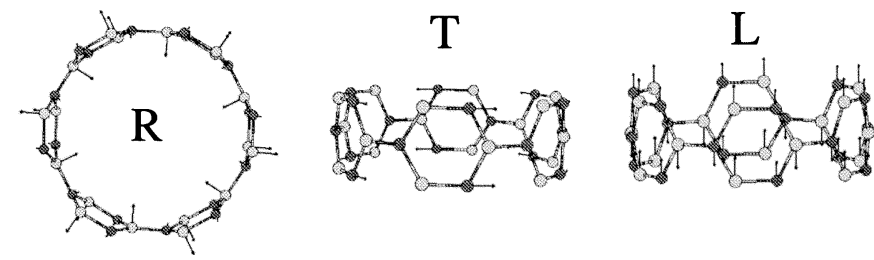

Fig. 5. High-frequency $B$ modes of a $(6,6)$ armchair nanotube.

Fig. 5 displays the $B$ modes of a $(6,6)$ armchair tube, which are derived from the $M$ point of the three optical phonon branches of the sheet. As in the case of the $A$ modes, the $\mathrm{R}$ and $\mathrm{T}$ modes have odd parity and the L mode has even parity. However, the behavior under rotation is different from the $A$ modes. The modes are mapped onto themselves under rotation by $\phi=2 \pi / n$ (corresponding to the symmetry group of the unit cell), but they change the direction of atomic motion under the basic symmetry operation of the nonsymmorphic rod group, which is a rotation by $\phi / 2$ accompanied by translation along the tube axis.

The central result of our ab initio calculations is the diameter dependence of the frequencies of Raman and IR active modes, which is displayed in Fig. 6 for armchair and zigzag tubes. In the right-hand-side panel, we also display the phonon dispersion relation of the single sheet in order to illustrate the zone-folding procedure. A detailed discussion of the results can be found in [26]. Here, we just give a summary of the most important features. The phonon spectrum can roughly be divided into three regimes. The phonons in the low-frequency region are derived from the acoustic branches of the sheet dispersion relation. With increasing diameter $d$, the phonon frequencies decrease as $1 / d$ or $1 / d^{2}$ corresponding to the liner/quadratic slope of the longitudinal acoustic (LA), transverse acoustic (TA), and out-of-plane acoustic (ZA) branches of the sheet dispersion, respectively. It is this strong diameter dependence that makes the low-frequency modes, especially the radial breathing mode, prime candidates for the diameter determination via Raman and IR STS. The phonons in the intermediate-frequency region around $800 \mathrm{~cm}^{-1}$ are derived from the optical out-of-plane (ZO) branch of the sheet. For large diameter, all three phonon modes $\left(A, E_{1}\right.$, and $E_{2}$ ) converge toward the frequency of the $\mathrm{ZO}$ mode at gamma. This frequency is strongly present in the IR spectra of multiwall $\mathrm{BN}$ tubes [24]. The high-frequency regime (above 1200 $\mathrm{cm}^{-1}$ comprises the phonon modes derived from the LO and TO branches of the sheet. The L modes converge monotonously increasing toward the asymptotic value at $1382 \mathrm{~cm}^{-1}$, while the $\mathrm{T}$ modes display a strong nonmonotonic behavior and approach the asymptotic value from above, corresponding to the strong over-bending of the LO branch in the dispersion relation of the sheet.

\section{RAman AND IR ACtive Modes IN TubeS OF FINITE LENGTHS}

If the tubes are shorter than the wavelength of incident laser light, the tubes appear as long molecules rather than as infinitely extended systems. In this section, we discuss the implication of the finite length for the number of Raman and IR active modes. 


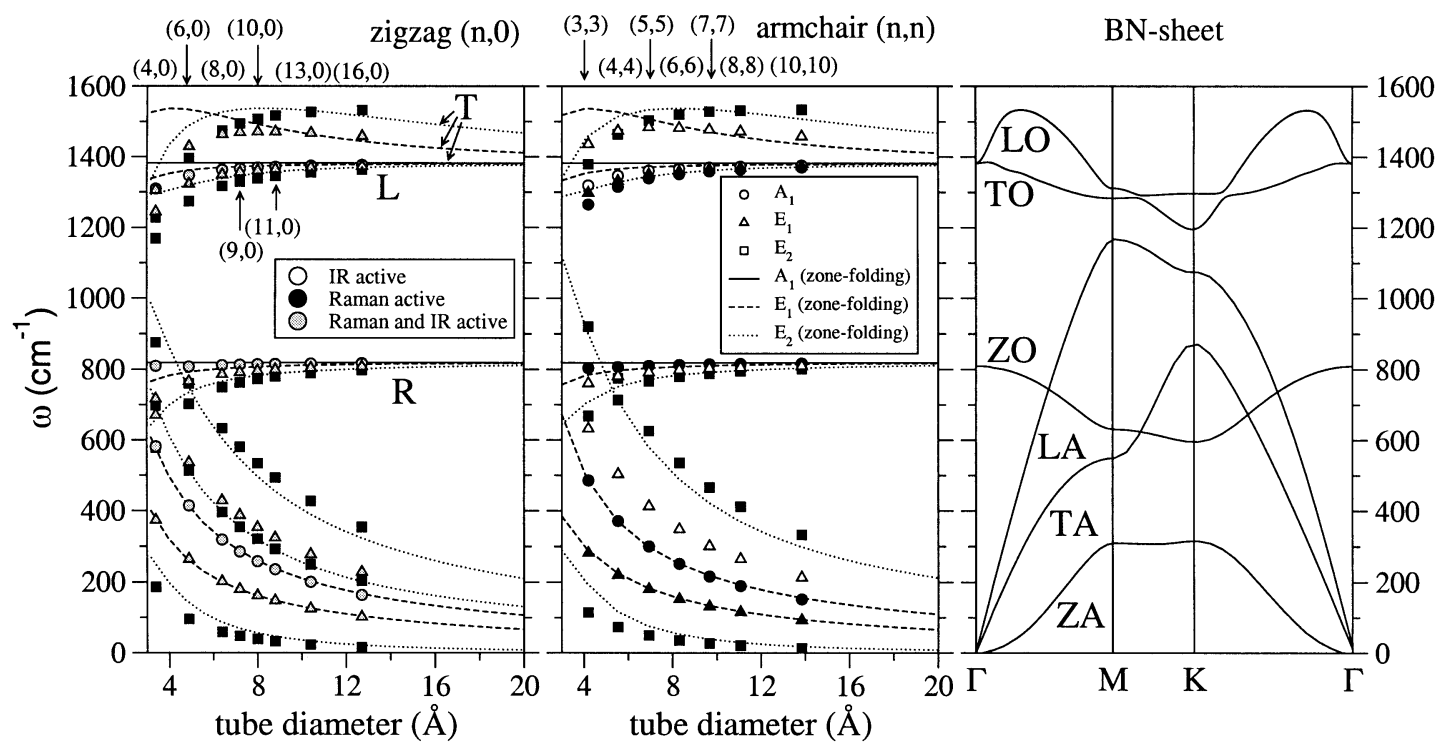

Fig. 6. Phonon frequencies of Raman and IR active modes of infinitely long zigzag and armchair BN nanotubes as a function of the tube diameter. The lines denote the result of the zone-folding method (compare to the dispersion relation of the single sheet in the right-hand-side panel). The symbols are the result of $a b$ initio calculations for selected tubes. The shape of the symbols characterizes its symmetry (see legend in the inset) and the shading in black, white, or gray distinguishes between modes that are Raman active, IR active, or both Raman and IR active, respectively.

TABLE II

SYMMETRIES AND NUMBER OF RAMAN AND IR ACTIVE MODES IN FINITE LENGTH AARMCHAIR AND ZIGZAG BN NANOTUBES. (NUMBER OF CENTRAL FREQUENCIES, i.e., FOR MODES WITH LONG WAVELENGTH ALONG THE TUBE AXIS.)

\begin{tabular}{|c|c|c|c|c|c|c|c|c|c|c|}
\hline & \multicolumn{8}{|c|}{ armchair $(n, n)$} & \multirow{3}{*}{\multicolumn{2}{|c|}{$\begin{array}{c}\text { zigzag }(n, 0) \\
C_{n v}\end{array}$}} \\
\hline & \multicolumn{4}{|c|}{$\begin{array}{c}C_{n h} \\
\text { (even number of unit cells) }\end{array}$} & \multicolumn{4}{|c|}{$\begin{array}{c}S_{2 n} \\
\text { (odd number of unit cells) }\end{array}$} & & \\
\hline & $n$ ev & & & dd & $n$ eve & & $n$ & & & \\
\hline \multirow[t]{3}{*}{$\overline{\overline{\text { Raman }}}$} & $\overline{\bar{A} A_{g}}$ & $\overline{77}$ & $\overline{A^{\prime}}$ & $\overline{7}$ & $\overline{\bar{A}}$ & $\overline{5}$ & $\overline{A_{g}}$ & $\overline{5}$ & $\overline{A_{1}}$ & 7 \\
\hline & $E_{1 g}$ & 4 & $E 1^{\prime \prime}$ & 4 & $E_{(n-1)}$ & 6 & $E_{1 g}$ & 6 & $E_{1}$ & 11 \\
\hline & $E_{2 g}$ & 8 & $E 2^{\prime}$ & 8 & $E_{2}$ & 6 & $E_{2 g}$ & 6 & $E_{2}$ & 12 \\
\hline \multirow[t]{2}{*}{$\overline{\mathrm{IR}}$} & $A_{u}$ & 3 & $A^{\prime \prime}$ & 3 & $B$ & 5 & $A_{u}$ & 5 & $A_{1}$ & 7 \\
\hline & $E_{1 u}$ & 7 & $E 1^{\prime}$ & 7 & $E_{1}$ & 5 & $E_{1 u}$ & 5 & $E_{1}$ & 11 \\
\hline
\end{tabular}

For the symmetry analysis, we assume that the ends of the tubes are terminated in a symmetric way. ${ }^{2}$ The symmetry analysis of chiral and zigzag tubes is unaffected by a nonsymmetric termination. However, the destruction of inversion symmetry/horizontal-reflection symmetry for armchair tubes eliminates the distinction between modes of even and odd parity in that case. For zigzag and chiral nanotubes, the finite length reduces the symmetry of the tube to the point-group symmetry of the unit cell (assuming that the tube ends do not destroy the point-group symmetry). For $(n, 0)$ zigzag tubes, this means that the symmetry group is $C_{n v}$, and for $(n, m)$ chiral tubes, this means that the symmetry group is $C_{d}$, where $d$ is the greatest common divisor between $n$ and $m, \operatorname{gcd}(n, m)$. The case of $(n, n)$ armchair tubes is a little bit more complicated: For an integer number of unit cells in the finite-length tube, the horizontal reflection symmetry of the unit cell is preserved and the symmetry group is $C_{n h}$. If the tube contains a half-integer number of unit cells, the reflection symmetry is lost, but the presence of improper rotations enforces the $S_{2 n}$ symmetry of the system. Table II sum-

${ }^{2}$ Note that, in BN systems, the formation of $\mathrm{B}-\mathrm{B}$ or $\mathrm{N}-\mathrm{N}$ bonds is avoided, which precludes termination of tubes by fullerene-like half-spheres, which would contain pentagon rings with two borons or nitrogens as nearest neighbors [42]. In samples of $\mathrm{BN}$ tubes, it was observed that the termination proceeds usually by a flat angular cap or with an encapsulated particle [17]. marizes the symmetry groups and the number of active modes for zigzag and armchair tubes of finite lengths. Here, we have omitted the case of chiral tubes. If $\operatorname{gcd}(n, m)=1$, in principle, all phonon modes can be active. However, the intensities of most of these modes may be vanishingly small. We note that the symmetry evaluation using the point group symmetry of the unit cell corresponds to the symmetry evaluation, which has been mostly employed for the symmetry evaluation of carbon tubes (up to the very recent accurate $a b$ initio calculations in [33]). In the zone-folding picture, the use of the point-group symmetry of the unit cell signifies that not only the modes of the $\Gamma$-point of the sheet, but also the modes of the $M$ point are mapped onto $A$ modes of the tube. This is indicated in Fig. 2 by the letters in parentheses. Furthermore, the modes close to the $M$ point, which would fold onto modes of $E_{n-1}$ and $E_{n-2}$ symmetry according to the strict symmetry assignment, posses $E_{1}$ and $E_{2}$ symmetry according to the more relaxed point-group symmetry of the unit cell.

The diameter dependence of all active modes in finite-length tubes is displayed in Fig. 7. Indeed, in comparison with Fig. 6, additional branches, which converge toward the phonons of the sheet at the $M$ point with frequencies $305,550,636,1168$, 1283 , and $1315 \mathrm{~cm}^{-1}$. Note the pronounced difference in the scaling with diameter of the additional branches between armchair and zigzag tubes. For example, the branch that approaches $1168 \mathrm{~cm}^{-1}$ is very flat in the case of the zigzag tubes, but has a strong diameter dependence for the armchair tubes. This is easily understood when keeping in mind that, for zigzag tubes, the zone folding is done along $\Gamma \rightarrow K \rightarrow M \rightarrow K \rightarrow \Gamma$ in the Brillouin zone of the sheet. This means that, in the dispersion relation of the sheet (see right-hand-side panel of Fig. 6), the $M$ point at $1168 \mathrm{~cm}^{-1}$ is approached from the right-hand side where the dispersion relation is very flat. For armchair tubes, the $M$ point is accordingly approached from the left-hand side where the dispersion relation is steep. In the case of the phonon 


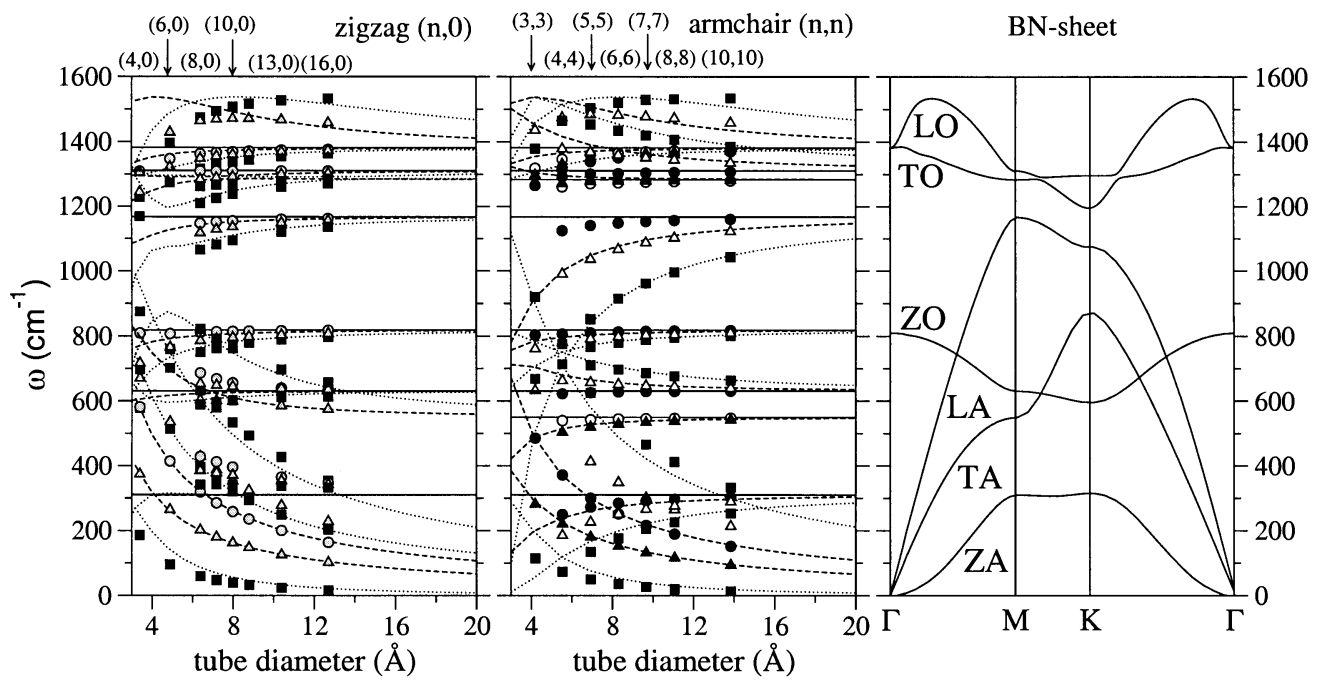

Fig. 7. Raman and IR active modes of BN nanotubes with finite lengths. For an explanation of the symbols, see Fig. 6.

branch approaching $550 \mathrm{~cm}^{-1}$ for $d \rightarrow \infty$, this effect is even stronger and leads to a monotonously decreasing convergence toward this value for zigzag tubes and to a monotonously increasing convergence for armchair tubes.

For the proposed frequencies of active modes in tubes of finite length in Fig. 7, we have assumed that the tubes are sufficiently long such that their frequencies closely resemble the frequencies of the infinitely long tube. In other words, we have used the phonons modes at $\Gamma$ of the infinitely long tube and performed the symmetry analysis as if the tubes had finite length. This approximation is justified for vibrational modes with very long wavelength along the tube axis. Modes with short wavelength along the tube axis have considerably different frequencies, which resemble the frequencies of phonons with nonvanishing $q$ in infinite tubes. However, the Raman and IR intensities are vanishingly small because the strong oscillations lead to very weak coupling-matrix elements with the laser field. Furthermore, the fact that a mode is "allowed" by the selection rules does not mean that it has a high intensity. The intensity of the modes that are allowed in finite tubes, but forbidden in infinitely long tubes, decays with the length of the tube. If the exact scaling is calculated, the intensities of these peaks can be used to determine the tube length.

\section{Phonon Frequencies in a Closely Packed Periodic ARRAY OF TUBES}

Since, in real samples [17], nanotubes occur both isolated and in bundles, it is important to investigate the effect of inter-tube interaction onto the phonon frequencies. For carbon nanotubes, only a weak perturbation was found for closely packed nanotube arrays. In the calculations of Kahn and Lu [43], the effect of bundling does not exceed $10 \mathrm{~cm}^{-1}$, except for the very low energy $E_{1}$ and $E_{2}$ modes. For the radial breathing mode (RBM), they found a stiffening by $4 \%$. Henrard et al. [44] found a value of $10 \%$ for the stiffening of the RBM due to bundling.

We investigate the effect of bundling in BN nanotubes by calculating a closely packed lattice of nanotubes with an inter-tube distance of 6.5 a.u., which corresponds approximately to the
TABLE III

Change of Phonon Frequencies (IN $\mathrm{cm}^{-1}$ ) DUE to Close PACKING OF TUBES IN A PERIODIC ARRAY: RAMAN AND IR ACTIVE MODES FOR AN ISOLATED BN $(8,0)$ TUBE (With AN INTERTUBE DisTANCE OF 14 a.u.) in COMParison With the Modes FOR A "Closely PACKeD" ARray OF TUbes (INTERTUBE DistANCE 6.5 a.u.). IF THE COUPLing TO AN ElECTRIC FIELd Is INCLUDED, A STRONG SHIFT/SPLITTING OF SOME Frequencies Is OBSERVED. (THE Numbers IN PARANTHESES ARE THE CORRESPONDING FREQUENCIES WITHOUT E-FIELD COUPLING)

\begin{tabular}{l|c|c|c}
\hline \multicolumn{4}{|c}{ Without coupling to macroscopic E-field } \\
\hline & & isolated BN(8,0) & close packed BN(8,0) \\
\hline$E_{2}$ & $\mathrm{R}$ & 59.3 & $66.5 / 76.5$ \\
$E_{1}$ & $\mathrm{~L}$ & 201.4 & $200.2 / 201.0$ \\
$A_{1}$ & $\mathrm{R}$ & 317.9 & 318.1 \\
$E_{2}$ & $\mathrm{~L}$ & 396.0 & $395.6 / 396.0$ \\
$E_{1}$ & $\mathrm{~T}$ & 428.4 & $422.3 / 431.0$ \\
$E_{2}$ & $\mathrm{~T}$ & 632.8 & $630.3 / 632.6$ \\
$E_{2}$ & $\mathrm{R}$ & 749.8 & $746.8 / 747.2$ \\
$E_{1}$ & $\mathrm{R}$ & 785.5 & $773.9 / 775.5$ \\
$A_{1}$ & $\mathrm{R}$ & 808.5 & 805.7 \\
$E_{1}$ & $\mathrm{~L}$ & 1348.9 & $1346.8 / 1347.1$ \\
$A_{1}$ & $\mathrm{~L}$ & 1362.5 & 1361.4 \\
$E_{1}$ & $\mathrm{~T}$ & 1463.4 & $1416.6 / 1417.8$ \\
$E_{2}$ & $\mathrm{~T}$ & 1474.0 & $1465.1 / 1470.4$ \\
\hline \hline \multicolumn{3}{|c|}{ E-field coupling with $q \rightarrow 0$ along tube axis } \\
\hline \multicolumn{5}{|c|}{} & isolated BN(8,0) & close packed BN(8,0) \\
\hline$A_{1}$ & $\mathrm{R}$ & $318.5(317.9)$ & $318.7(318.1)$ \\
$A_{1}$ & $\mathrm{R}$ & $811.6(808.5)$ & $809.0(805.6)$ \\
$A_{1}$ & $\mathrm{~L}$ & $1547.2(1362.5)$ & $1585.9(1361.5)$ \\
\hline \hline
\end{tabular}

inter-sheet distance in h-BN. As in the case of "isolated" tubes, the geometry is optimized, which leads to a slight deformation from the ideal cylindrical geometry and requires the displacement of all atoms in the unit cell for the calculation of phonon frequencies. In the upper part of Table III, we compare the (Raman and IR active) phonon frequencies of a closely packed $(8,0)$ tube with a (quasi-)isolated $(8,0)$ tube. The general effect of bundling is a slight shift of the nondegenerate $A$ modes and a splitting of the doubly degenerate $E$ modes into 


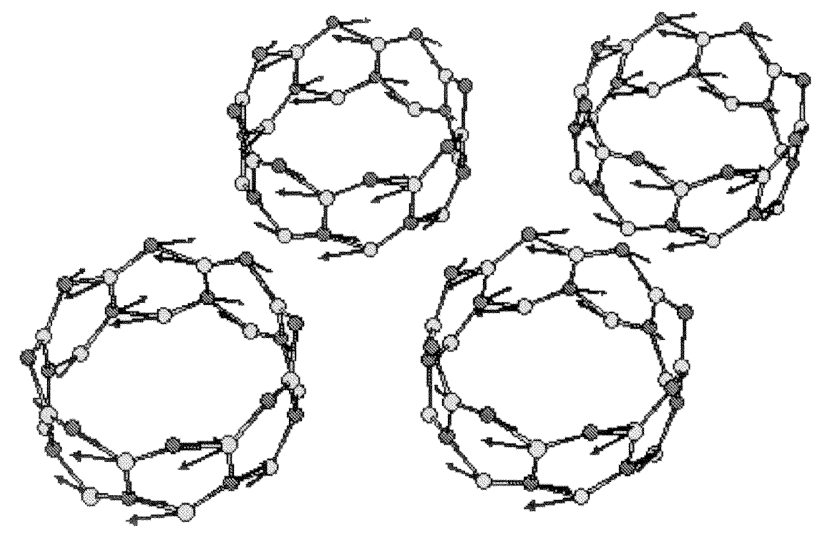

Fig. 8. High-frequency tangential $E_{1}$ mode in a closely packed array of $(8,0)$ BN nanotubes.

two modes with slightly different frequencies. Most phonon modes (except for the lowest $E_{2 g}$ mode) are softened, contrary to the observations in $\mathrm{C}$ nanotubes, where, on average, a slight stiffening of the modes was observed [43], [44]. Apparently, the electrostatic inter-tube interaction acts, on average, as an attractive force, which counteracts the hardening of phonons that would take place if only the repulsion caused by the beginning inter-tube overlap of the wave functions would modify the frequency. The effect, however, is weak and does not exceed $10 \mathrm{~cm}^{-1}$ in most cases. A notable exception to the weak influence of bundling is the high-frequency tangential (T) $E_{1}$ mode, which is softened by almost $50 \mathrm{~cm}^{-1}$. This mode is depicted in Fig. 8. At the distance of the closest approach between neighboring tubes, equivalent atoms are locally moving, as in two parallel planes. This softens the mode and makes it susceptible to LO-TO splitting in macroscopically extended nanotube arrays, as shown below. In $\mathrm{C}$ nanotubes, where the electrostatic interaction is absent, this $E_{1}$ modes do not experience a different shift by bundling than the $A$ and $E_{2}$ modes.

In macroscopic polar crystals, the longitudinal optical phonons give rise to an electric field, which leads to a shift in frequency and to a splitting of degenerate LO-TO modes. For bulk h-BN, this LO-TO splitting amounts to almost $200 \mathrm{~cm}^{-1}$ ([6]). "Macroscopic" in this context means that the dimensions of the "crystal" in all three directions are larger than the wavelength of the interacting laser light. While this is certainly not the case for nanotube bundles containing only tens of nanotubes, it is conceivable that, in the near future, better purification and alignment methods lead to large periodic arrays of nanotubes. In this case, the effect of coupling to the $E$-field will become important for the LO modes. In the following, LO always denotes longitudinal with respect to the wave vector of the interacting laser light. Even though the excited phonons close to $\Gamma$ carry approximately zero momentum, it does play a role from which direction the limit $\vec{q} \rightarrow 0$ is taken. Table III demonstrates that, for the laser light parallel to the tube axis, the $A_{1}$ modes experience a shift. In the case of the radial breathing and buckling modes, the shift is weak. However for the high-frequency $A_{1}$ (L) mode, the shift even exceeds the $200 \mathrm{~cm}^{-1}$ of the LO-TO splitting in h-BN. Note that the shift is also present for the periodic array of tubes with large inter-tube distance. Due to the long-range electrostatic interactions between the tubes, the shift decreases very slowly as a function of inter-tube distance. For incidence of the laser light perpendicular to the tube axis, it is the $E_{1}$ modes that experience a splitting in frequency. While all $E_{1}$ modes are slightly affected, it is the $E_{1}(\mathrm{~T})$ mode of Fig. 8 that experiences the relatively strong splitting of approximately $90 \mathrm{~cm}^{-1}$ in the closely packed array and $40 \mathrm{~cm}^{-1}$ in the array with large inter-tube distance. It can be seen from Fig. 8 that all B atoms are moving parallel and the $\mathrm{N}$ atoms likewise. This makes it a longitudinal optical mode in the macroscopic array of tubes with the laser light incident in the direction of oscillation. In anisotropic polar systems, such as h-BN, the directional dependence of the LO-TO splitting has been used for angular resolved STS (ellipsometry) [4]. Due to the variety of modes, macroscopic arrays of $\mathrm{BN}$ nanotubes promise to be an even richer playground for angular resolved IR spectroscopy.

\section{CONCLUSION}

Since BN nanotubes can now be produced in macroscopic (i.e., gram) quantities, Raman and IR spectroscopy will very likely develop into standard tools for characterization of the tubes according to diameter, length, and chirality. The characterization requires accurate data on the vibrational properties of the tubes. We have presented $a b$ initio calculations of the phonon frequencies as a function of tube diameter and tube chirality. The phonon modes display a very close similarity to the phonon modes in carbon tubes. However, due to the reduced rod group symmetry, more modes are Raman and IR active. We have discussed possible deviations from the phonon frequencies of ideal, isolated, and infinitely long tubes in realistic samples of BN tubes. The finite length of the tube (typically shorter than the wavelength of IR light) leads to a reduction of the tube symmetry and thereby to additional active modes. The Raman and IR intensity of these modes might serve as an indicator for the average length of the measured tubes. An important difference with respect to carbon tubes is the polarity of BN. In large arrays of closely packed tubes, the polarity leads to a coupling of longitudinal optical modes with the internal electric field. The corresponding frequency shift of these modes depends on the direction of the incoming light and the average distance between the tubes. The richness of predicted phonon frequencies gives rise to the hope that, in the future, large samples of clean and well-aligned samples can be produced and spectroscopically investigated.

\section{ACKNOWLEDGMENT}

The authors acknowledge stimulating discussions with F. Mauri, M. Lazzeri, R. Arenal de la Concha, and A. Loiseau. The authors are particularly grateful to O. Alon for his explanations on the symmetry analysis of nanotubes and for careful reading of the manuscript. A portion of this paper's calculations was performed at CEPBA, Barcelona, Spain.

\section{REFERENCES}

[1] R. Geick, C. H. Perry, and G. Rupprecht, "Normal modes in hexagonal boron nitride," Phys. Rev., vol. 146, pp. 543-547, June 1966. 
[2] D. M. Hoffmann, G. L. Doll, and P. C. Eklund, "Optical properties of pyrolitic boron nitride in the energy range $0.05-10 \mathrm{eV}$," Phys. Rev. B, Condens. Matter, vol. 30, pp. 6051-6056, Nov. 1984.

[3] X. Blase, A. Rubio, S. G. Louie, and M. L. Cohen, "Quasiparticle band structure of bulk hexagonal boron nitride and related systems," Phys. Rev. B, Condens. Matter, vol. 51, pp. 6868-6875, Mar. 1995.

[4] M. Schubert, B. Rheinländer, E. Franke, H. Neumann, T. E. Tiwald, J. A. Woollam, J. Hahn, and F. Richter, "Infrared optical properties of mixed-phase thin films studied by spectroscopic ellipsometry using boron nitride as an example," Phys. Rev. B, Condens. Matter, vol. 56, pp. 13 306-13 313, Nov. 1997.

[5] E. Rokuta, Y. Hasagawa, K. Suzuki, Y. Gamou, C. Oshima, and A. Nashima, "Phonon dispersion of an epitaxial monolayer film of hexagonal boron nitride on $\mathrm{Ni}(111)$," Phys. Rev. Lett., vol. 79, pp. 4609-4612, Dec. 1997

[6] G. Kern, G. Kresse, and J. Hafner, "Ab initio calculation of the lattice dynamics and phase diagram of boron nitride," Phys. Rev. B, Condens. Matter, vol. 59, pp. 8551-8559, Apr. 1999

[7] A. Rubio, J. L. Corkill, and M. L. Cohen, "Theory of graphitic boron nitride nanotubes," Phys. Rev. B, Condens. Matter, vol. 49, pp. 5081-5084, Feb. 1994.

[8] X. Blase, A. Rubio, S. G. Louie, and M. L. Cohen, "Stability and band gap constancy of boron nitride nanotubes," Europhys. Lett., vol. 28, pp. 335-340, Nov. 1994

[9] N. G. Chopra, J. Luyken, K. Cherry, V. H. Crespi, M. L. Cohen, S. G. Louie, and A. Zettl, "Boron nitride nanotubes," Science, vol. 269, pp. 966-967, Aug. 1995.

[10] A. Loiseau, F. Willaime, N. Demoncy, G. Hung, and H. Pascard, "Boron nitride nanotubes with reduced numbers of layers synthesized by arc discharge," Phys. Rev. Lett., vol. 76, pp. 4737-4740, June 1996.

[11] D. P. Yu, X. S. Sun, C. S. Lee, I. Bello, S. T. Lee, H. D. Gu, K. M. Leung, G. W. Zhou, Z. F. Dong, and Z. Zhang, "Synthesis of boron nitride nanotubes by means of excimer laser ablation at high temperature," Appl. Phys. Lett., vol. 72, pp. 1966-1968, Apr. 1998.

[12] D. Goldberg, Y. Bando, W. Han, K. Kurashima, and T. Sato, "Singlewalled B-doped carbon, B/N-doped carbon and BN nanotubes synthesized from single-walled carbon nanotubes through a substitution reaction," Chem. Phys. Lett., vol. 308, pp. 337-342, July 1999.

[13] Y. Chen, J. F. Gerald, J. S. Williams, and S. Bulcock, "Synthesis of boron nitride nanotubes at low temperatures using reactive ball milling," Chem. Phys. Lett., vol. 299, pp. 260-264, Jan. 1999.

[14] J. Cummings and A. Zettl, "Mass-production of boron nitride double-wall nanotubes and nanococoons," Chem. Phys. Lett., vol. 316, pp. 211-216, Jan. 2000.

[15] T. Laude, Y. Matsui, A. Marraud, and B. Jouffrey, "Long ropes of boron nitride nanotubes grown by a continuous laser heating," Appl. Phys. Lett., vol. 76, pp. 3239-3241, May 2000

[16] E. Bengu and L. D. Marks, "Single-walled BN nanostructures," Phys. Rev. Lett., vol. 86, pp. 2385-2387, Mar. 2001.

[17] R. S. Lee, J. Gavillet, M. L. de la Chapelle, A. Loiseau, J.-L. Cochon, D. Pigache, J. Thibault, and F. Willaime, "Catalyst-free synthesis of boron nitride single-wall nanotubes with a preferred zigzag configuration," Phys. Rev. B, Condens. Matter, vol. 64, Sept. 2001.

[18] L. C. Venema, J. W. Janssen, M. R. Buitelaar, J. W. G. Wildöer, S. G. Lemay, L. P. Kouwenhoven, and C. Dekker, "Spatially resolved scanning tunneling spectroscopy on single-walled carbon nanotubes," Phys. Rev. B, Condens. Matter, vol. 62, pp. 5238-5244, Aug. 2000.

[19] G. G. Fuentes, E. Borowiak-Palen, T. Pichler, X. Liu, A. Graff, G. Behr, R. J. Kalenczuk, M. Knuper, and J. Fink, "Electronic structure of multiwall boron nitride nanotubes," Phys. Rev. B, Condens. Matter, vol. 67, Jan. 2003.

[20] S. M. Bachilo, M. S. Strano, C. Kittrell, R. H. Hauge, R. E. Smalley, and R. B. Weisman, "Structure-assigned optical spectra of single-walled carbon nanotubes," Sciene, vol. 298, pp. 2361-2366, Nov. 2003.

[21] A. M. Rao, E. Richter, S. Bandow, B. Chase, P. C. Eklund, K. A. Williams, S. Fang, K. R. Subbaswamy, M. Menon, A. Thess, R. E. Smalley, G. Dresselhaus, and M. S. Dresselhaus, "Diameter-selective Raman scattering from vibrational modes in carbon nanotubes," Science, vol. 275, pp. 187-191, Jan. 1997.

[22] R. Saito, T. Takeya, T. Kimura, G. Dresselhaus, and M. S. Dresselhaus, "Raman intensity of single-wall carbon nanotubes," Phys. Rev. B, Condens. Matter, vol. 57, pp. 4145-4153, Feb. 1998.
[23] R. A. de la Concha, L. Wirtz, J.-Y. Mevellec, S. Lefrant, A. Loiseau, and A. Rubio, . unpublished.

[24] E. Borowiak-Palen, T. Pichler, G. G. Fuentes, B. Bendjemil, X. Liu, A. Graff, G. Behr, R. J. Kalenczuk, M. Knupfer, and J. Fink, "Infrared response of multiwalled boron nitride nanotubes," Chem. Commun., pp. 82-83, 2003.

[25] U. Kuhlmann, H. Jontoljak, N. Pfänder, P. Bernier, C. Journet, and C. Thomsen, "Infrared active phonons in single-walled carbon nanotubes," Chem. Phys. Lett., vol. 294, pp. 237-240, Sept. 1998.

[26] L. Wirtz, A. Rubio, R. A. de la Concha, and A. Loiseau, "Ab initio calculations of the lattice dynamics of boron nitride nanotubes," Phys. Rev. B, Condens. Matter, vol. 68, July 2003.

[27] O. E. Alon, "Symmetry properties of single-walled boron nitride nanotubes," Phys. Rev. B, Condens. Matter, vol. 64, Oct. 2001.

[28] M. Damnjanović, T. Vuković, I. Miloŝević, and B. Nikolić, "Symmetry of single-wall nanotubes," Act. Cryst., vol. A57, pp. 304-310, 2001.

[29] O. E. Alon, "From spatial symmetry to vibrational spectroscopy of single-walled nanotubes," J. Phys., Condens. Matter, vol. 15, pp. 2489-2500, Sept. 2003, to be published.

[30] N. W. Ashcroft and N. D. Mermin, Solid State Physics. Orlando, FL: Saunders College, 1976.

[31] R. Saito, G. Dresselhaus, and M. S. Dresselhaus, Physical Properties of Carbon Nanotubes. London, U.K.: Imperial College Press, 1998.

[32] R. A. Jishi, L. Venkataraman, M. S. Dresselhaus, and G. Dresselhaus, "Phonon modes in carbon nanotubules," Chem. Phys. Lett., vol. 209, pp. 77-82, June 1993.

[33] O. Dubay and G. Kresse, "Accurate density functional calculations for the phonon dispersion relations of graphite layer and carbon nanotubes," Phys. Rev. B, Condens. Matter, vol. 67, Jan. 2003.

[34] J. Yu, R. K. Kalia, and P. Vashishta, "Phonons in graphitic tubules: A tight-binding molecular dynamics study," J. Chem. Phys., vol. 103, pp. 6697-6705, Oct. 1995.

[35] D. Sánchez-Portal, E. Artacho, J. M. Soler, A. Rubio, and P. Ordejón, "Ab initio structural, elastic, and vibrational properties of carbon nanotubes," Phys. Rev. B, Condens. Matter, vol. 59, pp. 12 678-12 688, May 1999.

[36] D. Sánchez-Portal and E. Hernández, "Vibrational properties of single-wall nanotubes and monolayers of hexagonal BN," Phys. Rev. B, Condens. Matter, vol. 66, Dec. 2002.

[37] V. N. Popov, "Lattice dynamics of single-walled boron nitride nanotubes," Phys. Rev. B, Condens. Matter, vol. 67, pp. ???-???, Feb. 2003.

[38] N. Troullier and J. L. Martins, "Efficient pseudopotentials for plane-wave calculations," Phys. Rev. B, Condens. Matter, vol. 43, pp. 1993-2006, Jan. 1991.

[39] S. Baroni, S. de Gironcoli, A. Dal Corso, and P. Giannozzi, "Phonons and related crystal properties from density-functional perturbation theory," Rev. Mod. Phys., vol. 73, pp. 515-562, Apr. 2001.

[40] X. Gonze and C. Lee, "Dynamical matrices, born effective charges, dielectric permittivity tensors, and interatomic force constants from density-functional perturbation theory," Phys. Rev. B, Condens. Matter, vol. 55, pp. 10355-10368, Apr. 1997.

[41] W. Kohn and L. J. Sham, "Self-consistent equations including exchange and correlation effects," Phys. Rev., vol. 140, pp. A1133-A1138, Nov. 1965.

[42] G. Seifert, P. W. Fowler, D. Mitchell, D. Porezag, and T. Frauenheim, "Boron-nitrogen analogues of the fullerenes: Electronic and structural properties," Chem. Phys. Lett., vol. 268, pp. 352-358, Apr. 1997.

[43] D. Kahn and J. P. Lu, "Vibrational modes of carbon nanotubes and nanoropes," Phys. Rev. B, Condens. Matter, vol. 60, pp. 6535-6540, Sept. 1999.

[44] L. Henrard, E. Hernández, P. Bernier, and A. Rubio, "Van der Waals interaction in nanotube bundles: Consequences on vibrational modes," Phys. Rev. B, Condens. Matter, vol. 60, pp. 8521-8524, Sept. 1999.

Ludger Wirtz, photograph and biography not available at time of publication.

Angel Rubio, photograph and biography not available at time of publication. 